\title{
Lie Symmetry Analysis, Conservation Laws, Power Series Solutions, and Convergence Analysis of Time Fractional Generalized Drinfeld-Sokolov Systems
}

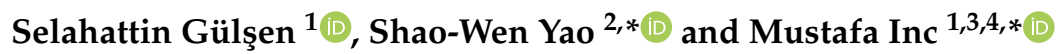 \\ 1 Department of Mathematics, Faculty of Science, Firat University, Elazig 23119, Turkey; \\ sgulsen2301@gmail.com \\ 2 School of Mathematics and Information Science, Henan Polytechnic University, Jiaozuo 454000, China \\ 3 Department of Computer Engineering, Biruni University, Istanbul 34096, Turkey \\ 4 Department of Medical Research, China Medical University Hospital, China Medical University, \\ Taichung 40402, Taiwan \\ * Correspondence: yaoshaowen@hpu.edu.cn (S.-W.Y.); minc@firat.edu.tr (M.I.)
}

Citation: Gülşen, S.; Yao, S.-W.; Inc,

M. Lie Symmetry Analysis,

Conservation Laws, Power Series

Solutions, and Convergence Analysis of Time Fractional Generalized Drinfeld-Sokolov Systems. Symmetry 2021, 13, 874. https://doi.org/ $10.3390 /$ sym 13050874

Academic Editor: Sergei D. Odintsov

Received: 23 March 2021

Accepted: 6 May 2021

Published: 14 May 2021

Publisher's Note: MDPI stays neutral with regard to jurisdictional claims in published maps and institutional affiliations.

Copyright: (C) 2021 by the authors. Licensee MDPI, Basel, Switzerland. This article is an open access article distributed under the terms and conditions of the Creative Commons Attribution (CC BY) license (https:// creativecommons.org/licenses/by/ $4.0 /)$.

\begin{abstract}
In this work, we investigate invariance analysis, conservation laws, and exact power series solutions of time fractional generalized Drinfeld-Sokolov systems (GDSS) using Lie group analysis. Using Lie point symmetries and the Erdelyi-Kober (EK) fractional differential operator, the time fractional GDSS equation is reduced to a nonlinear ordinary differential equation (ODE) of fractional order. Moreover, we have constructed conservation laws for time fractional GDSS and obtained explicit power series solutions of the reduced nonlinear ODEs that converge. Lastly, some figures are presented for explicit solutions.
\end{abstract}

Keywords: conservation laws; Lie symmetry analysis; generalized Drinfeld-Sokolov systems; symmetry reduction; Erdelyi-Kober operators

\section{Introduction}

Because of the great importance of nonlinear fractional partial differential equations (NFPDEs) in physics, mechanics, hydrology, viscoelasticity, image processing, electromagnetics, and other fields, researchers have long been aware of the solutions and applications of fractional partial differential equations [1-12]. In recent years, parallel to the increase in mathematical techniques and the use of computer programs, many authors have an increased desire to work on fractional analysis. Therefore, many methods are used to solve the NFPDEs-for example, the finite-difference method [13], the multiple exp-function method [14], the homotopy perturbation method [15,16], the variational-iteration method, the exp method [17], the fractional sub-equation method [18], and the Lie invariance method [19-21]. When we look through the literature, we realize Sophus Lie firstly put forward a methodology about symmetry analysis at the end of the nineteenth century [22]. After that, some impressive Lie group methods were considered in order to obtain symmetries, symmetry groups, and symmetry reduction. These are the classical and nonclasical Lie group approaches [23,24] and the Clarkson and Kruksal direct methods [25,26]. The main role of Lie symmetry methods is to construct invariance properties having partial equations as invariant forms. With the aid of these properties, we can reduce an NFPDE into a nonlinear ODE of fractional order with the help of the Riemann-Liouville (RL) derivative.

The link between Lie symmetry analysis and conservation laws of differential equations was revealed by Noether [27]. A generalized Noether theorem was used in [28] to construct conservation laws of NFPDEs with fractional Lagrangians. However, some differential equations do not arise from Lagrangians. To overcome this problem, İbragimov [29] put forward a new method without Lagrangians. Lukashchuk [30] showed important results towards obtaining conservation laws of NFPDEs. 
In this study, we deal with exact solutions of the time fractional GDSS by using Lie symmetry analysis and conservation laws. The time fractional GDSS that models 1D nonlinear wave processes in two-component media has the form

$$
\begin{aligned}
& D_{t}^{\alpha} u+a_{1} u u_{x}+b_{1} u_{x x x}+c\left(v^{q}\right)_{x}=0, \\
& D_{t}^{\alpha} v+a_{2} u v_{x}+b_{2} v_{x x x}=0,
\end{aligned}
$$

where $a_{1}, a_{2}, b_{1}, b_{2}, c$, and $q$ are arbitrary constants, and $D_{t}^{\alpha}$ is the RL fractional derivative defined in (2). We could not take $b_{1}=b_{2}=0$, because the time fractional GDSS may develop finite time singularities [31-33]. According to our research, Lie symmetry methods have not been applied to the time fractional GDSS until now. With some special choices of $a_{1}$, $a_{2}, b_{1}, b_{2}, c$, and $q$, the equation can be reduced to the time fractional Drinfeld-SokolovSatsuma-Hirota equations discussed in [34].

The RL fractional partial derivative [35] is defined by

$$
D_{t}^{\alpha} u= \begin{cases}\frac{\partial^{m} u}{\partial^{t}}, & \alpha=m \in N, \\ \frac{1}{\Gamma(m-\alpha)} \frac{\partial^{m}}{\partial t^{m}} \int_{0}^{t} \frac{u(z, x)}{(t-z)^{(\alpha+1-m)}} d z, & m-1<\alpha<m, \quad m \in N\end{cases}
$$

where $\Gamma(z)$ is a Gamma function defined by

$$
\Gamma(z)=\int_{0}^{\infty} e^{-t} t^{z-1} d t
$$

which converges in the complex plane when $\operatorname{Re}(z)>0$.

\section{Preliminaries for Symmetry Analysis}

In this chapter, we give the basic idea of the Lie symmetry method. Consider a system of time fractional partial differential equations with independent variables $x$ and $t$ as follows:

$$
\begin{aligned}
& D_{t}^{\alpha} u(x, t)=F_{1}\left(x, t, u, u_{t}, u_{x}, u_{x x}, v, v_{t}, v_{x}, v_{x x, \ldots}\right), \\
& D_{t}^{\alpha} v(x, t)=F_{2}\left(x, t, u, u_{t}, u_{x}, u_{x x}, v, v_{t}, v_{x}, v_{x x, \ldots}\right),
\end{aligned}
$$

where the subscripts denote partial derivatives. Equations (3) and (4) are invariant under a one-parameter Lie group of point transformations given as follows:

$$
\begin{array}{lrl}
\bar{t}=t+\epsilon \tau(t, x, u, v)+O\left(\epsilon^{2}\right), & \bar{x}=x+\epsilon \zeta(t, x, u, v)+O\left(\epsilon^{2}\right), \\
\bar{u}=u+\epsilon \mu(t, x, u, v)+O\left(\epsilon^{2}\right), & \bar{v}=v+\epsilon \rho(t, x, u, v)+O\left(\epsilon^{2}\right), \\
\frac{\partial^{\alpha} \bar{u}}{\partial \bar{t}^{\alpha}}=\frac{\partial^{\alpha} u}{\partial t^{\alpha}}+\epsilon \mu_{\alpha}^{0}(t, x, u, v)+O\left(\epsilon^{2}\right), & \frac{\partial^{\alpha} \bar{v}}{\partial \bar{t}^{\alpha}}=\frac{\partial^{\alpha} v}{\partial t^{\alpha}}+\epsilon \rho_{\alpha}^{0}(t, x, u, v)+O\left(\epsilon^{2}\right), \\
\frac{\partial \bar{u}}{\partial \bar{x}}=\frac{\partial u}{\partial x}+\epsilon \mu^{x}(t, x, u, v)+O\left(\epsilon^{2}\right), & \frac{\partial^{2} \bar{u}}{\partial \bar{x}^{2}}=\frac{\partial^{2} u}{\partial x^{2}}+\epsilon \mu^{x x}(t, x, u, v)+O\left(\epsilon^{2}\right), \\
\frac{\partial^{3} \bar{u}}{\partial \bar{x}^{3}}=\frac{\partial^{3} u}{\partial x^{3}}+\epsilon \mu^{x x x}(t, x, u, v)+O\left(\epsilon^{2}\right), & \frac{\partial \bar{v}}{\partial \bar{x}}=\frac{\partial v}{\partial x}+\epsilon \rho^{x}(t, x, u, v)+O\left(\epsilon^{2}\right), \\
\frac{\partial^{2} \bar{v}}{\partial \bar{x}^{2}}=\frac{\partial^{2} v}{\partial x^{2}}+\epsilon \rho^{x x}(t, x, u, v)+O\left(\epsilon^{2}\right), & \frac{\partial^{3} \bar{v}}{\partial \bar{x}^{3}}=\frac{\partial^{3} v}{\partial x^{3}}+\epsilon \rho^{x x x}(t, x, u, v)+O\left(\epsilon^{2}\right),
\end{array}
$$


where

$$
\begin{aligned}
& \mu^{x}=D_{x}(\mu)-u_{x} D_{x}(\zeta)-u_{t} D_{x}(\tau), \\
& \mu^{x x}=D_{x}\left(\mu^{x}\right)-u_{x x} D_{x}(\zeta)-u_{x t} D_{x}(\tau), \\
& \mu^{x x x}=D_{x}\left(\mu^{x x}\right)-u_{x x x} D_{x}(\zeta)-u_{x x t} D_{x}(\tau), \\
& \rho^{x}=D_{x}(\rho)-v_{x} D_{x}(\zeta)-v_{t} D_{x}(\tau), \\
& \rho^{x x}=D_{x}\left(\rho^{x}\right)-v_{x x} D_{x}(\zeta)-v_{x t} D_{x}(\tau), \\
& \rho^{x x x}=D_{x}\left(\rho^{x x}\right)-v_{x x x} D_{x}(\zeta)-v_{x x} D_{x}(\tau), \\
& \mu_{\alpha}^{0}=D_{t}^{\alpha}(\mu)+\zeta D_{t}^{\alpha}\left(u_{x}\right)-D_{t}^{\alpha}\left(\zeta u_{x}\right)+D_{t}^{\alpha}\left(u\left(D_{t} \rho\right)\right)-D_{t}^{\alpha+1}(\rho u)+\rho D_{t}^{\alpha+1}(u), \\
& \rho_{\alpha}^{0}=D_{t}^{\alpha}(\rho)+\zeta D_{t}^{\alpha}\left(v_{x}\right)-D_{t}^{\alpha}\left(\zeta v_{x}\right)+D_{t}^{\alpha}\left(v\left(D_{t} \rho\right)\right)-D_{t}^{\alpha+1}(\rho v)+\rho D_{t}^{\alpha+1}(v),
\end{aligned}
$$

wherein $D_{x}$ and $D_{t}$ are total differential operators given as follows:

$$
\begin{aligned}
& D_{x}=\frac{\partial}{\partial x}+u_{x} \frac{\partial}{\partial u}+u_{x x} \frac{\partial}{\partial u_{x}}+v_{x} \frac{\partial}{\partial v}+v_{x x} \frac{\partial}{\partial v_{x}}+\ldots, \\
& D_{t}=\frac{\partial}{\partial t}+u_{t} \frac{\partial}{\partial u}+u_{t t} \frac{\partial}{\partial u_{t}}+v_{t} \frac{\partial}{\partial v}+v_{t t} \frac{\partial}{\partial v_{t}}+\ldots
\end{aligned}
$$

The infinitesimal generator of Equations (3) and (4) consists of a set of vector fields given by

$$
X=\zeta \frac{\partial}{\partial x}+\tau \frac{\partial}{\partial t}+\mu \frac{\partial}{\partial u}+\rho \frac{\partial}{\partial v} .
$$

One can prove that the infinitesimal generators defined above must satisfy the following invariance criterion for Equations (3) and (4):

$$
\left.\operatorname{Pr}^{n} X\left(\Delta_{1}\right)\right|_{\Delta_{1}=0}=0 \text { and }\left.\operatorname{Pr}^{n} X\left(\Delta_{2}\right)\right|_{\Delta_{2}=0}=0, \quad n=1,2, . . .
$$

where

$$
\Delta_{1}:=D_{t}^{\alpha} u(x, t)-F_{1}\left(x, t, u, u_{t}, u_{x}, u_{x x}, v, v_{t}, v_{x}, v_{x x}, \ldots\right),
$$

and

$$
\Delta_{2}:=D_{t}^{\alpha} v(x, t)-F_{2}\left(x, t, u, u_{t}, u_{x}, u_{x x}, v, v_{t}, v_{x}, v_{x x, \ldots}\right) .
$$

As the lower limit of integral in (1) is fixed, it is going to be invariant under the transformations given in (5), so the corresponding invariance condition becomes

$$
\tau(t, x, u, v)=0 .
$$

Under the condition (9), the $\alpha$-th infinitesimal related to RL fractional time derivative [36] is given by

$$
\begin{aligned}
& \mu_{\alpha}^{0}=\frac{\partial^{\alpha} \mu}{\partial t^{\alpha}}+\left(\mu_{u}-\alpha D_{t}(\tau)\right) \frac{\partial^{\alpha} u}{\partial t^{\alpha}}-u \frac{\partial^{\alpha} \mu_{u}}{\partial t^{\alpha}}-v \frac{\partial^{\alpha} \mu_{v}}{\partial t^{\alpha}}+\mu_{v} \frac{\partial^{\alpha} v}{\partial t^{\alpha}}-\sum_{n=1}^{\infty}\left(\begin{array}{l}
\alpha \\
n
\end{array}\right) D_{t}^{n}(\zeta) D_{t}^{\alpha-n}\left(u_{x}\right) \\
& +\sum_{n=1}^{\infty}\left[\left(\begin{array}{c}
\alpha \\
n
\end{array}\right) \frac{\partial^{n} \mu_{u}}{\partial t^{n}}-\left(\begin{array}{c}
\alpha \\
n+1
\end{array}\right) D_{t}^{n+1}(\tau)\right] D_{t}^{\alpha-n}(u)+\sum_{n=1}^{\infty}\left(\begin{array}{c}
\alpha \\
n
\end{array}\right) \frac{\partial^{n} \mu_{v}}{\partial t^{n}} D_{t}^{\alpha-n}(v)+\lambda, \\
& \rho_{\alpha}^{0}=\frac{\partial^{\alpha} \rho}{\partial t^{\alpha}}+\left(\rho_{v}-\alpha D_{t}(\tau)\right) \frac{\partial^{\alpha} v}{\partial t^{\alpha}}-u \frac{\partial^{\alpha} \rho_{u}}{\partial t^{\alpha}}-v \frac{\partial^{\alpha} \rho_{v}}{\partial t^{\alpha}}+\rho_{u} \frac{\partial^{\alpha} u}{\partial t^{\alpha}}-\sum_{n=1}^{\infty}\left(\begin{array}{l}
\alpha \\
n
\end{array}\right) D_{t}^{n}(\zeta) D_{t}^{\alpha-n}\left(v_{x}\right) \\
& +\sum_{n=1}^{\infty}\left[\left(\begin{array}{l}
\alpha \\
n
\end{array}\right) \frac{\partial^{n} \rho_{v}}{\partial t^{n}}-\left(\begin{array}{c}
\alpha \\
n+1
\end{array}\right) D_{t}^{n+1}(\tau)\right] D_{t}^{\alpha-n}(v)+\sum_{n=1}^{\infty}\left(\begin{array}{l}
\alpha \\
n
\end{array}\right) \frac{\partial^{n} \rho_{u}}{\partial t^{n}} D_{t}^{\alpha-n}(u)+\beta,
\end{aligned}
$$


where

$$
\begin{aligned}
& \lambda=\sum_{n=2}^{\infty} \sum_{m_{1}+m_{2}=2}^{n} \sum_{k_{1}=0}^{m_{1}} \sum_{r_{1}=0}^{k_{1}} \sum_{k_{2}=0}^{m_{2}} \sum_{r_{2}=0}^{k_{2}}\left(\begin{array}{c}
\alpha \\
n
\end{array}\right)\left(\begin{array}{c}
n \\
m_{1}
\end{array}\right)\left(\begin{array}{c}
n-m_{1} \\
m_{2}
\end{array}\right)\left(\begin{array}{l}
k_{1} \\
r_{1}
\end{array}\right)\left(\begin{array}{c}
k_{2} \\
r_{2}
\end{array}\right) \frac{1}{k_{1} ! k_{2} !} \frac{t^{n-\alpha}}{\Gamma(n+1-\alpha)} \\
& \times(-u)^{r_{1}}(-v)^{r_{2}} \frac{\partial^{m_{1}} u^{k_{1}-r_{1}}}{t^{m_{1}}} \frac{\partial^{m_{2}} v^{k_{2}-r_{2}}}{t^{m_{2}}} \frac{\partial^{n-m_{1}-m_{2}+k_{1}+k_{2}} \mu}{\partial^{n-m_{1}-m_{2} \partial u^{k_{1}} \partial v^{k_{2}}}}
\end{aligned}
$$

and

$$
\begin{aligned}
& \beta=\sum_{n=2}^{\infty} \sum_{m_{1}+m_{2}=2}^{n} \sum_{k_{1}=0}^{m_{1}} \sum_{r_{1}=0}^{k_{1}} \sum_{k_{2}=0}^{m_{2}} \sum_{r_{2}=0}^{k_{2}}\left(\begin{array}{c}
\alpha \\
n
\end{array}\right)\left(\begin{array}{c}
n \\
m_{1}
\end{array}\right)\left(\begin{array}{c}
n-m_{1} \\
m_{2}
\end{array}\right)\left(\begin{array}{c}
k_{1} \\
r_{1}
\end{array}\right)\left(\begin{array}{c}
k_{2} \\
r_{2}
\end{array}\right) \frac{1}{k_{1} ! k_{2} !} \frac{t^{n-\alpha}}{\Gamma(n+1-\alpha)} \\
& \times(-u)^{r_{1}}(-v)^{r_{2}} \frac{\partial^{m_{1}} u^{k_{1}-r_{1}}}{t^{m_{1}}} \frac{\partial^{m_{2}} v^{k_{2}-r_{2}}}{t^{m_{2}}} \frac{\partial^{n-m_{1}-m_{2}+k_{1}+k_{2}} \rho}{\partial^{n-m_{1}-m_{2}} \partial u^{k_{1}} \partial v^{k_{2}}} .
\end{aligned}
$$

Theorem 1. $u=v_{1}(x, t)$ and $v=v_{2}(x, t)$ are invariant solutions of Equations (3) and (4) if and only if

(i) $u=v_{1}(x, t)$ and $v=v_{2}(x, t)$ satisfy the following expressions:

$$
X v_{1}=0 \Leftrightarrow\left(\zeta \frac{\partial}{\partial x}+\tau \frac{\partial}{\partial t}+\mu \frac{\partial}{\partial u}+\rho \frac{\partial}{\partial v}\right) v_{1}=0
$$

and

$$
X v_{2}=0 \Leftrightarrow\left(\zeta \frac{\partial}{\partial x}+\tau \frac{\partial}{\partial t}+\mu \frac{\partial}{\partial u}+\rho \frac{\partial}{\partial v}\right) v_{2}=0
$$

(ii) $u=v_{1}(x, t)$ and $v=v_{2}(x, t)$ are also solutions of (3) and (4), respectively.

\section{Lie Symmetry Analysis and Reduction of Time Fractional GDSS}

In this part of the work, we acquire an infinitesimal generator of the Generalized Drinfeld-Sokolov systems by applying Lie point symmetries. Assume that Equation (1) are invariant under one parameter transformations (5). We then have that

$$
\begin{aligned}
& \bar{u}_{\bar{t}}^{\alpha}+a_{1} \bar{u} \bar{u}_{\bar{x}}+b_{1} \bar{u}_{\bar{x} \bar{x} \bar{x}}+c\left(\bar{v}^{q}\right)_{\bar{x}}=0, \\
& \bar{v}_{\bar{t}}^{\alpha}+a_{2} \bar{u}_{\bar{v}} \bar{v}_{\bar{x}}+b_{2} \bar{v}_{\bar{x} \bar{x} \bar{x}}=0 .
\end{aligned}
$$

Applying the second prolongation $P r^{2}$ transformation to Equation (1), then using transformation (6), we obtain invariant equations as follows:

$$
\begin{aligned}
& \mu_{\alpha}^{0}+a_{1} u_{x} \mu+a_{1} u \mu^{x}+b_{1} \mu^{x x x}+c q(q-1) v^{q-2} \rho v_{x}=0, \\
& \rho_{\alpha}^{0}+a_{2} v_{x} \mu+a_{2} u \rho^{x}+c \rho^{x x x x}=0 .
\end{aligned}
$$

Putting the values of $\rho_{\alpha}^{0}, \mu_{\alpha}^{0}, \rho, \mu \mu^{x}, \mu^{x x x}, \rho^{x}$, and $\rho^{x x x}$ from Equation (6) into Equation (12) and then isolating the coefficients in partial derivatives with respect to $u$ and $v$, we obtain a determined system of linear equations stated as 


$$
\begin{aligned}
& \mu_{u u}=\rho_{v v}=\zeta_{u}=\tau_{u}=\tau_{t}=\tau_{x}=\zeta_{u}=\tau_{v}=\tau_{x}=\mu_{v}=\tau_{x u}=\rho_{u u u}=\ldots=\mu_{x v u}=0, \\
& \alpha \tau_{t}+3 \mu x=0 \text {, } \\
& -a_{1} u \tau_{v}+\alpha c q v^{q-1} \tau_{u}-3 b_{1} \tau_{x x v}=0, \\
& \alpha a_{1} u \tau_{t}+c v^{q-1} q \rho_{u}-a_{1} u \zeta_{x}=0, \\
& -a_{2} u \zeta_{x}+a_{2} \mu-b_{2} \zeta_{x x x}+3 b_{2} \rho_{x x v}+\alpha \tau_{t}=0, \\
& \left(\begin{array}{l}
\alpha \\
n
\end{array}\right)\left(\frac{\partial^{n} \rho_{v}}{\partial t^{n}}\right) D_{t^{\alpha-n}} v-\frac{1}{n+1}\left(\begin{array}{l}
\alpha \\
n
\end{array}\right)\left[D_{t^{\alpha-n}} v D_{t^{1+n}} \alpha \tau-D_{t^{\alpha-n}} v D_{t^{1+n}} n \tau\right. \\
& \left.+D_{t^{\alpha-n}} v_{x} D_{t^{n}} n \zeta+D_{t^{\alpha-n}} v_{x} D_{t^{n}} \zeta\right]=0 . \quad n=3,4, \ldots .
\end{aligned}
$$

Solving all these determination equations, we obtain an explicit form of infinitesimal symmetry for Equation (1) as

$$
\zeta=-c_{1} \alpha q x+c_{2}, \quad \tau=-3 t q c_{1}, \quad \mu=2 u q c_{1} \alpha, \quad \rho=4 v \alpha c_{1},
$$

where $c_{1}$ and $c_{2}$ are arbitrary constants. Thus, we can construct corresponding vector fields:

$$
X=\left(-c_{1} \alpha q x+c_{2}\right) \frac{\partial}{\partial x}-3 t q c_{1} \frac{\partial}{\partial t}+2 u q \alpha c_{1} \frac{\partial}{\partial u}+4 v \alpha c_{1} \frac{\partial}{\partial v} .
$$

It is stated that there are two vector fields spanning Equation (1):

$$
X_{1}=\frac{\partial}{\partial x}, \quad X_{2}=-\alpha q x \frac{\partial}{\partial x}-3 t q \frac{\partial}{\partial t}+2 u q \alpha \frac{\partial}{\partial u}+4 v \alpha \frac{\partial}{\partial v} .
$$

Case 1. For the symmetry $X_{1}$, we can write characteristics equations as follows:

$$
\frac{d x}{1}=\frac{d t}{0}=\frac{d u}{0}=\frac{d v}{0}
$$

Solving these characteristic equations, we can easily obtain a trivial solution.

Case 2. Lastly we focus on symmetry $X_{2}$, so we can write characteristic equations as

$$
\frac{d x}{-\alpha q x}=\frac{d t}{-3 q t}=\frac{d u}{2 q \alpha u}=\frac{d v}{4 v \alpha} .
$$

Solving these characteristic equations, we get both the similarity variable and the similarity transformation as follows:

$$
\xi=x t^{\frac{-\alpha}{3}}, \quad u=t^{\frac{-2 \alpha}{3}} f(\xi), \quad v=t^{\frac{-4 \alpha}{3 q}} g(\xi),
$$

where $f$ and $g$ are arbitrary functions of $\xi$.

Theorem 2. The similarity transformations (15) reduce Equation (1) to a nonlinear ODE with a fractional order of the form

$$
\begin{aligned}
& \left(P_{\frac{3}{\alpha}}^{1-\frac{5 \alpha}{3}, \alpha} f\right)(\xi)+a_{1} f f_{\xi}+b_{1} f_{\xi \xi \xi}+c q g^{q-1} g_{\xi}=0, \\
& \left(P_{\frac{3}{\alpha}}^{1-\alpha-\frac{4 \alpha}{3 q}, \alpha} g\right)(\xi)+a_{2} f g_{\xi}+b_{2} g_{\xi \xi \xi}=0,
\end{aligned}
$$


where the Erdelyi-Kober (EK) fractional differential operators [37] are given as

$$
\begin{aligned}
& \left(P_{\beta}^{\tau, \alpha} f\right)(\xi):=\prod_{j=0}^{n-1}\left(\tau+j-\frac{1}{\beta} \xi \frac{d}{d \xi}\right)\left(K_{\beta}^{\tau+\alpha, n-\alpha} f\right)(\xi), \\
& \left(P_{\beta}^{\tau, \alpha} g\right)(\xi):=\prod_{j=0}^{n-1}\left(\tau+j-\frac{1}{\beta} \xi \frac{d}{d \xi}\right)\left(K_{\beta}^{\tau+\alpha, n-\alpha} g\right)(\xi),
\end{aligned}
$$

and with the EK Fractional integral operators $[38,39]$ defined as

$$
\begin{gathered}
\left(K_{\beta}^{\tau+\alpha, n-\alpha} f\right)(\xi):=\left\{\begin{array}{cc}
\frac{1}{\Gamma(\alpha)} \int_{1}^{\infty}(u-1)^{\alpha-1} u^{-(\tau+\alpha)} f\left(\xi \mathcal{u}^{\frac{1}{\beta}}\right) d u, & \alpha>0, \\
f(\xi), & \alpha=0,
\end{array}\right. \\
\left(K_{\beta}^{\tau+\alpha, n-\alpha} g\right)(\xi):=\left\{\begin{array}{cc}
\frac{1}{\Gamma(\alpha)} \int_{1}^{\infty}(u-1)^{\alpha-1} u^{-(\tau+\alpha)} g\left(\xi \mathcal{u}^{\frac{1}{\beta}}\right) d u, & \alpha>0, \\
g(\xi), & \alpha=0,
\end{array}\right.
\end{gathered}
$$

and

$$
n= \begin{cases}{[\alpha]+1,} & \alpha \in N, \\ \alpha, & \alpha \notin N .\end{cases}
$$

Proof. Let $n-1<\alpha<n, n=1,2,3, \ldots$. Using the transformations defined in (15) and the definition of the RL fractional derivative, we have

$$
D_{t}^{\alpha} u(x, t)=\frac{\partial n}{\partial t^{n}}\left[\frac{1}{\Gamma(n-\alpha)} \int_{0}^{t}(t-s)^{n-\alpha-1} s^{\frac{-2 \alpha}{3}} f\left(x s^{\frac{-\alpha}{3}}\right) d s\right] .
$$

Let $v=\frac{t}{s}$, so $d s=\frac{-t}{v^{2}} d v$. Equation (22) becomes

$$
\begin{gathered}
D_{t}^{\alpha} u(x, t)=\frac{\partial n}{\partial t^{n}}\left[\frac{1}{\Gamma(n-\alpha)} \int_{1}^{\infty} t^{n-\frac{5 \alpha}{3}}(v-1)^{n-1-\alpha} v^{n+1-\frac{5 \alpha}{3}} f\left(\xi^{\frac{\alpha}{3}}\right) d v\right], \\
D_{t}^{\alpha} u(x, t)=\frac{\partial n}{\partial t^{n}}\left[t^{n-\frac{5 \alpha}{3}}\left(K_{\frac{3}{\alpha}}^{1-\frac{2 \alpha}{3}, n-\alpha} f\right)(\xi)\right], \\
D_{t}^{\alpha} u(x, t)=\frac{\partial n-1}{\partial t^{n-1}}\left[\frac{\partial}{\partial t}\left(t^{n-\frac{5 \alpha}{3}}\left(K_{\frac{3}{\alpha}}^{1-\frac{2 \alpha}{3}, n-\alpha} f\right)(\xi)\right)\right] \\
D_{t}^{\alpha} u(x, t)=\frac{\partial n-1}{\partial t^{n-1}}\left[\frac{\partial}{\partial t}\left(t^{n-1-\frac{5 \alpha}{3}}\left(n-\frac{5 \alpha}{3}-\frac{\alpha}{3} \xi \frac{d}{d \xi}\right)\left(K_{\frac{3}{\alpha}}^{1-\frac{2 \alpha}{3}, n-\alpha} f\right)(\xi)\right)\right] .
\end{gathered}
$$

Applying the above procedure $n-1$ times, we obtain

$$
D_{t}^{\alpha} u(x, t)=t^{-\frac{5 \alpha}{3}}\left(\prod_{j=0}^{n-1}\left(1-\frac{5 \alpha}{3}+j-\frac{\alpha}{3} \xi \frac{d}{d \xi}\right)\left(K_{\frac{3}{\alpha}}^{1-\frac{2 \alpha}{3}, n-\alpha} f\right)(\xi)\right) .
$$

Now, by using Equation (17), we can immediately write

$$
D_{t}^{\alpha} u(x, t)=\frac{\partial n}{\partial t^{n}}\left[t^{n-\frac{5 \alpha}{3}}\left(K_{\frac{3}{\alpha}}^{1-\frac{2 \alpha}{3}, n-\alpha} f\right)(\xi)\right]=t^{-\frac{5 \alpha}{3}}\left(P_{\frac{3}{\alpha}}^{1-\frac{5 \alpha}{3}, \alpha} f\right)(\xi) .
$$


Consequently, we prove that the first equation defined in (1) reduces to an ODE of a fractional order:

$$
\left(P_{\frac{3}{\alpha}}^{1-\frac{5 \alpha}{3}, \alpha} f\right)(\xi)+a_{1} f f_{\xi}+b_{1} f_{\xi \xi \xi}+c q g^{q-1} g_{\xi}=0 .
$$

Similarly, using transformations (15) and the definition of the RL fractional derivative, we easily reduced the second equation of (1) into an ODE:

$$
\left(P_{\frac{3}{\alpha}}^{1-\alpha-\frac{4 \alpha}{3 q}, \alpha} g\right)(\xi)+a_{2} f g_{\xi}+b_{2} g_{\xi \xi \xi}=0 .
$$

The proof is completed.

\section{Conservation Laws for the Time Fractional GDSS}

Before obtaining the conserved vector for the time fractional GDSS, some important definitions should be given. We can start with the RL right-sided time fractional derivative, defined as follows:

$$
o D_{t}^{n}\left(o I^{n-\alpha} f\right),
$$

where $D_{t}$ is a total differential operator with respect to $t$, and the $o I^{n-\alpha}$ is the right-sided time-fractional integral of $n-\alpha$ [40] given by

$$
o I^{n-\alpha} f(x, t)=\frac{1}{\Gamma(n-\alpha)} \int_{t}^{p} \frac{f(x, \theta)}{(\theta-t)^{1-n+\alpha}} d \theta,
$$

where $n=[\alpha]+1$.

All solutions of $u(x, t)$ and $v(x, t)$ provide the following conservation equation:

$$
D_{t}\left(N^{t}\right)+D_{x}\left(N^{x}\right)=0,
$$

where $N^{t}=N^{t}(x, t, u, .$.$) and N^{x}=N^{x}(x, t, u, .$.$) .$

We use the İbragimov method [29] to construct conservation laws of Equation (1). The formal Langrangian of Equation (1) is defined as the following:

$$
\mathcal{L}=\phi(x, t)\left(D_{t}^{\alpha} u+a_{1} u u_{x}+b_{1} u_{x x x}+c q v^{q-1} v_{x}\right)+\sigma(x, t)\left(D_{t}^{\alpha} v+a_{2} u v_{x}+b_{2} v_{x x x}\right),
$$

where $\sigma$ and $\phi$ define the new dependent variable of $x$ and $t$.

Now, we express Euler-Langrangian operators [41] as follows:

$$
\frac{\delta}{\delta u}=\frac{\partial}{\partial u}+\left(D_{t}^{\alpha}\right)^{*} \frac{\partial}{\partial D_{t}^{\alpha} u}-D_{x} \frac{\partial}{\partial u_{x}}-D_{x}^{3} \frac{\partial}{\partial u_{x x x}},
$$

and

$$
\frac{\delta}{\delta v}=\frac{\partial}{\partial v}+\left(D_{t}^{\alpha}\right)^{*} \frac{\partial}{\partial D_{t}^{\alpha} v}-D_{x} \frac{\partial}{\partial v_{x}}-D_{x}^{3} \frac{\partial}{\partial v_{x x x}}
$$

where $\left(D_{t}^{\alpha}\right)^{*}$ is the adjoint operator of $D_{t}^{\alpha}$. sion:

Using the Euler Lagrange Equations (35) and (36), we can write the following expres-

$$
\frac{\delta \mathcal{L}}{\delta u}=0, \quad \text { and } \quad \frac{\delta \mathcal{L}}{\delta v}=0 .
$$

Thus, we have

$$
\bar{X}+D_{t}(\tau) \mathcal{I}+D_{x}(\zeta) \mathcal{I}=W_{1} \frac{\delta}{\delta u}+W_{2} \frac{\delta}{\delta v}+D_{t} N^{t}+D_{x} N^{x},
$$


where $\mathcal{I}$ denotes the identity operator, $\frac{\delta}{\delta u}$ and $\frac{\delta}{\delta v}$ represents the Euler Lagrange operators, $C^{t}$ and $C^{x}$ are the Noether operators, and $\bar{X}$ is defined as

$$
\begin{aligned}
& \bar{X}=\zeta \frac{\partial}{\partial x}+\tau \frac{\partial}{\partial t}+\mu \frac{\partial}{\partial u}+\rho \frac{\partial}{\partial v}+\mu_{\alpha}^{0} \frac{\partial}{\partial D_{t}^{\alpha} u}+\rho_{\alpha}^{0} \frac{\partial}{\partial D_{t}^{\alpha} v}+\mu^{x} \frac{\partial}{\partial u_{x}} \\
& +\mu^{x x x} \frac{\partial}{\partial u_{x x x}}+\rho^{x} \frac{\partial}{\partial v_{x}}+\rho^{x x x} \frac{\partial}{\partial v_{x x x}},
\end{aligned}
$$

and we can give Lie characteristics functions $W$ for vector field $X_{2}$ as

$$
W_{1}=\mu-\tau u_{t}-\zeta u_{x}=2 u q \alpha+3 t q u_{t}-\alpha q x u_{x}
$$

and

$$
W_{2}=\rho-\tau v_{t}-\zeta v_{x}=4 v \alpha+3 t q v_{t}-\alpha q x v_{x} .
$$

Using the RL time fractional derivative in Equation (1), we now write components of conserved vectors [40,41], as follows:

$$
\begin{aligned}
& N^{t}=\tau \mathcal{I}+\sum_{k=0}^{n-1} o D_{t}^{\alpha-1-k}\left(W_{1}\right) D_{t}^{k} \frac{\partial \mathcal{L}}{\partial o D_{t}^{\alpha} u}-(-1)^{n} J\left(W 1, D_{t}^{n} \frac{\partial \mathcal{L}}{\partial o D_{t}^{\alpha} u}\right) \\
& +\sum_{k=0}^{n-1} o D_{t}^{\alpha-1-k}\left(W_{2}\right) D_{t}^{k} \frac{\partial \mathcal{L}}{\partial o D_{t}^{\alpha} v}-(-1)^{n} J\left(W 2, D_{t}^{n} \frac{\partial \mathcal{L}}{\partial o D_{t}^{\alpha} v}\right)
\end{aligned}
$$

where $J$ is defined as follows:

$$
J(r, s)=\frac{1}{\Gamma(n-1)} \int_{0}^{t} \int_{t}^{T} \frac{r(\tau, x) s(v, x)}{(v-\tau)^{\alpha+1-n}} d v d t,
$$

and the explicit form of $N^{x}$ for Equation (1) is given as

$$
\begin{aligned}
& N^{x}=\zeta \mathcal{I}+W_{1}\left[\frac{\partial}{\partial u_{x}}-D_{x} \frac{\partial}{\partial u_{x x}}+D_{x} D_{x} \frac{\partial}{\partial u_{x x x}}\right]+W_{2}\left[\frac{\partial}{\partial v_{x}}-D_{x} \frac{\partial}{\partial v_{x x}}+D_{x} D_{x} \frac{\partial}{\partial v_{x x x}}\right] \\
& +D_{x}\left(W_{1}\right)\left[\frac{\partial}{\partial u_{x x}}-D_{x} \frac{\partial}{\partial u_{x x x}}\right]+D_{x}\left(W_{2}\right)\left[\frac{\partial}{\partial v_{x x}}-D_{x} \frac{\partial}{\partial v_{x x x}}\right]+D_{x} D_{x}\left[\frac{\partial}{\partial u_{x x x}}\right] \\
& +D_{x} D_{x}\left[\frac{\partial}{\partial v_{x x x}}\right] .
\end{aligned}
$$

We can now derive the corresponding conserved vectors respectively as follows: i: $\quad \alpha \in(0,1)$

$$
\begin{aligned}
& N_{1}^{t}=\phi_{0} D_{t}^{\alpha-1}\left(2 u q \alpha+3 t q u_{t}-\alpha q x u_{x}\right)+J\left(\left(2 u q \alpha+3 t q u_{t}-\alpha q x u_{x}\right), \phi_{t}\right) \\
& +\sigma_{0} D_{t}^{\alpha-1}\left(4 v \alpha+3 t q u_{t}-\alpha q x v_{x}\right)+J\left(\left(4 v \alpha+3 t q v_{t}-\alpha q x v_{x}\right), \sigma_{t}\right),
\end{aligned}
$$

ii: $\quad \alpha \in(1,2)$

$$
\begin{aligned}
& N_{2}^{t}=\phi_{0} D_{t}^{\alpha-1}\left(2 u q \alpha+3 t q u_{t}-\alpha q x u_{x}\right)+J\left(\left(2 u q \alpha+3 t q u_{t}-\alpha q x u_{x}\right), \phi_{t}\right) \\
& -\phi_{t} o D_{t}^{\alpha-2}\left(2 u q \alpha+3 t q u_{t}-\alpha q x u_{x}\right)-J\left(\left(2 u q \alpha+3 t q u_{t}-\alpha q x u_{x}\right), \phi_{t t}\right) \\
& +\sigma_{0} D_{t}^{\alpha-1}\left(4 v \alpha+3 t q u_{t}-\alpha q x v_{x}\right)+J\left(\left(4 v \alpha+3 t q v_{t}-\alpha q x v_{x}\right), \sigma_{t}\right) \\
& -\sigma_{t} o D_{t}^{\alpha-2}\left(4 v \alpha+3 t q u_{t}-\alpha q x v_{x}\right)-J\left(\left(4 v \alpha+3 t q u_{t}-\alpha q x v_{x}\right), \sigma_{t t}\right),
\end{aligned}
$$


and

$$
\begin{aligned}
& N_{1}^{x}=W_{1}\left(\phi a_{1} u+b_{1} \phi_{x x}\right)+W_{2}\left(\phi c q v^{q-1}+\sigma a_{2} u+b_{2} \sigma_{x x}\right)-b_{1} \phi_{x} D_{x}\left(W_{1}\right) \\
& -b_{2} \sigma_{x} D_{x}\left(W_{2}\right)+b_{1} \phi D_{x}^{2}\left(W_{1}\right)+b_{2} \sigma D_{x}^{2}\left(W_{2}\right),
\end{aligned}
$$

where

$$
W_{1}=2 u q \alpha+3 t q u_{t}-\alpha q x u_{x} \text { and } W_{2}=4 v \alpha+3 t q v_{t}-\alpha q x v_{x} .
$$

\section{Series Solutions of Equations (29) and (30)}

In this section, we examine the power series solution of the system. This method is more accurate and efficient for obtaining an exact analytical solution. The procedures of the method are given in [42]. We can construct

$$
\begin{gathered}
f(\xi)=\sum_{n=0}^{\infty} r_{n} \xi^{n}, \quad g(\xi)=\sum_{n=0}^{\infty} s_{n} \xi^{n}, \\
f_{\xi}=\sum_{n=1}^{\infty} n r_{n} \xi^{n-1}, \quad f_{\xi \xi \xi}=\sum_{n=3}^{\infty} n(n-1)(n-2) r_{n} \xi^{n-3},
\end{gathered}
$$

and

$$
g_{\xi}=\sum_{n=1}^{\infty} n s_{n} \xi^{n-1}, \quad g_{\xi \xi \xi}=\sum_{n=3}^{\infty} n(n-1)(n-2) s_{n} \xi^{n-3} .
$$

Substituting Equations (44)-(46) into Equations (29) and (30), we have

$$
\begin{aligned}
& \sum_{n=0}^{\infty} \frac{\Gamma\left(2-\frac{2 \alpha}{3}+\frac{n \alpha}{3}\right)}{\Gamma\left(2-\frac{5 \alpha}{3}+\frac{n \alpha}{3}\right)} r_{n} \xi^{n}+a_{1} \sum_{n=0}^{\infty} r_{n} \xi^{n} \sum_{n=0}^{\infty}(n+1) r_{n+1} \xi^{n} \\
& +b_{1} \sum_{n=0}^{\infty}(n+3)(n+2)(n+1) r_{n+3} \xi^{n}+c q\left(\sum_{n=0}^{\infty} s_{n} \xi^{n}\right)^{q-1} \sum_{n=0}^{\infty}(n+1) s_{n+1} \xi^{n}=0, \\
& \text { and } \\
& \qquad \sum_{n=0}^{\infty} \frac{\Gamma\left(2-\frac{4 \alpha}{3 q}+\frac{n \alpha}{3}\right)}{\Gamma\left(2-\alpha-\frac{4 \alpha}{3 q}+\frac{n \alpha}{3}\right)} s_{n} \xi^{n}+a_{2} \sum_{n=0}^{\infty} r_{n} \xi^{n} \sum_{n=0}^{\infty}(n+1) s_{n+1} \xi^{n} \\
& +b_{2} \sum_{n=0}^{\infty}(n+3)(n+2)(n+1) s_{n+3} \xi^{n}=0 .
\end{aligned}
$$

When $n=0$ in Equations (47) and (48), we obtain the following coefficients:

$$
r_{3}=\frac{1}{6 b_{1}}\left(\frac{\Gamma\left(2-\frac{2 \alpha}{3}\right)}{\Gamma\left(2-\frac{5 \alpha}{3}\right)} r_{0}+a_{1} r_{0} r_{1}+c q s_{1}^{q-1} s_{0}\right),
$$

and

$$
s_{3}=\frac{1}{6 b_{2}}\left(\frac{\Gamma\left(2-\frac{4 \alpha}{3 q}\right)}{\Gamma\left(2-\alpha-\frac{4 \alpha}{3 q}\right)} s_{0}+a_{2} r_{0} s_{1}\right) .
$$

When $n \geqslant 1$, we have the following coefficients

$$
\begin{aligned}
& r_{n+3}=\frac{1}{b_{1}(n+3)(n+2)(n+1)}\left(\frac{\Gamma\left(2-\frac{2 \alpha}{3}+\frac{n \alpha}{3}\right)}{\Gamma\left(2-\frac{5 \alpha}{3}+\frac{n \alpha}{3}\right)} r_{n}+a_{1} \sum_{k=0}^{n}(n-k+1) r_{n-k+1} r_{k}\right. \\
& \left.+c q \sum_{k_{1}=0}^{n} \sum_{k_{2}=0}^{k_{1}} \ldots \sum_{k_{q-1}=0}^{k_{q-2}}(n-k+1) s_{n-k+1} s_{k_{1}-k_{2} \ldots s_{k_{q-2}}-k_{q-1} s_{k_{q-1}}}\right),
\end{aligned}
$$




$$
s_{n+3}=\frac{\text { and }}{b_{2}(n+3)(n+2)(n+1)}\left(\frac{\Gamma\left(2-\frac{4 \alpha}{3 q}+\frac{n \alpha}{3}\right)}{\Gamma\left(2-\alpha-\frac{4 \alpha}{3 q}+\frac{n \alpha}{3}\right)} s_{n}+a_{2} \sum_{k=0}^{n}(n-k+1) s_{n-k+1} r_{k}\right) .
$$

Thus, the coefficients $r_{n}(n \geqslant 1)$ and $s_{n}(n \geqslant 1)$ can be calculated for Equation (44) as arbitrary constants. This shows that there are power series solutions to the Equations (29) and (30). We then acquire the explicit power series solutions of Equation (1) (Figures 1 and 2) as

$$
\begin{aligned}
& u(x, t)=r_{0} t^{\frac{-2 \alpha}{3}}+r_{1} x t^{-\alpha}+r_{2} x^{2} t^{\frac{-4 \alpha}{3}}+\frac{1}{6 b_{1}}\left(\frac{\Gamma\left(2-\frac{2 \alpha}{3}\right)}{\Gamma\left(2-\frac{5 \alpha}{3}\right)} r_{0}+a_{1} r_{0} r_{1}+c q s_{0}^{q-1} s_{1}\right) x^{3} t^{\frac{-5 \alpha}{3}} \\
& +\sum_{n=1}^{\infty} \frac{1}{b_{1}(n+3)(n+2)(n+1)}\left(\frac{\Gamma\left(2-\frac{2 \alpha}{3}+\frac{n \alpha}{3}\right)}{\Gamma\left(2-\frac{5 \alpha}{3}+\frac{n \alpha}{3}\right)} r_{n}+a_{1} \sum_{k=0}^{n}(n-k+1) r_{n-k+1} r_{k}\right. \\
& \left.+c q \sum_{k_{1}=0}^{n} \sum_{k_{2}=0}^{k_{1}} \ldots \sum_{k_{q-1}=0}^{k_{q-2}}(n-k+1) s_{n-k+1} s_{k_{1}-k_{2}} \ldots s_{k_{q-2}-k_{q-1} s_{k_{q-1}}}\right) x^{n+3} t^{\frac{-\alpha(n+5)}{3}}, \\
& v(x, t)=s_{0} t^{\frac{-4 \alpha}{3 q}}+s_{1} x t^{\frac{-\alpha(q+4)}{3 q}}+s_{2} x^{2} t^{\frac{-\alpha(2 q+4)}{3 q}}+\frac{1}{6 b_{2}}\left(\frac{\Gamma\left(2-\frac{4 \alpha}{3 q}\right)}{\Gamma\left(2-\alpha-\frac{4 \alpha}{3 q}\right)} s_{0}+a_{2} r_{0} s_{1}\right) x^{3} t^{\frac{-\alpha(3 q+4)}{3 q}} \\
& +\sum_{n=1}^{\infty} \frac{1}{b_{2}(n+3)(n+2)(n+1)}\left(\frac{\Gamma\left(2-\frac{4 \alpha}{3 q}+\frac{n \alpha}{3}\right)}{\Gamma\left(2-\alpha-\frac{4 \alpha}{3 q}+\frac{n \alpha}{3}\right)} s_{n}+a_{2} \sum_{k=0}^{n}(n-k+1) s_{n-k+1} r_{k}\right) \\
& \times x^{n+3} t^{\frac{-\alpha(n q+3 q+4)}{3 q}} \text {. }
\end{aligned}
$$
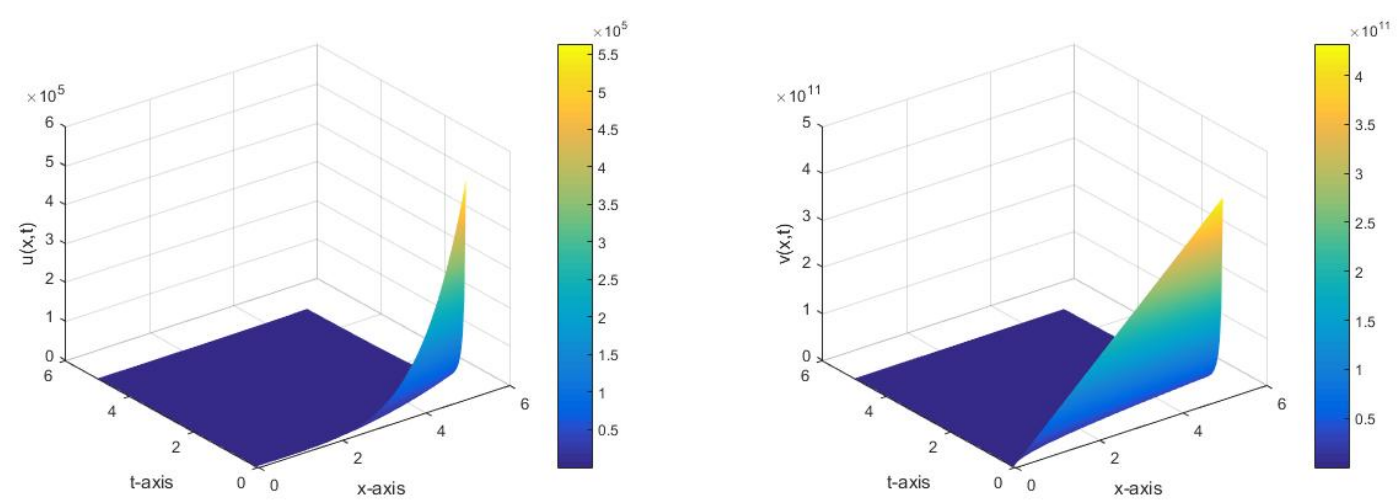

Figure 1. 3-D plots of Equations (53) and (54) with $r_{0}=1, r_{1}=2, r_{2}=3, s_{0}=s_{1}=s_{2}=2, b_{1}=b_{2}=1, a_{1}=a_{2}=2, q=2$, $c=2$ and $\alpha=0.5$. 

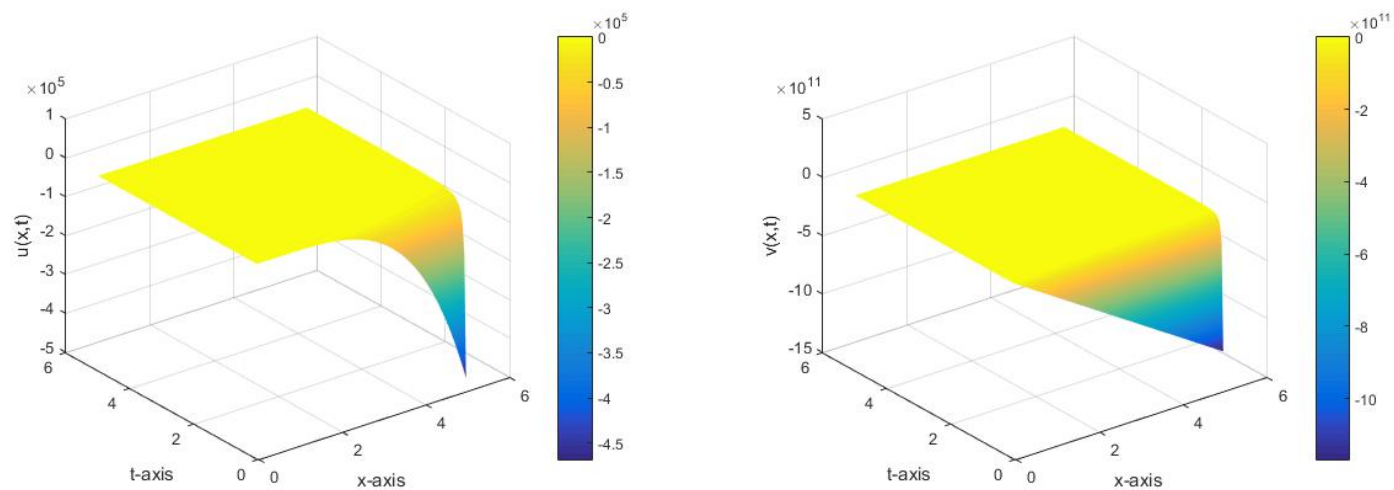

Figure 2. 3-D plots of Equations (53) and (54) with $r_{0}=2, r_{1}=2, r_{2}=2, s_{0}=s_{1}=s_{2}=1, b_{1}=1, b_{2}=6, a_{1}=-6$, $a_{2}=-2, q=1, c=-6$ and $\alpha=0.75$.

\section{Convergence Analysis of the Power Series Solution}

In this part of the work, we will prove that the power series solutions (53) and (54) are convergent. Considering Equations (51) and (52), we can write

$$
\begin{aligned}
& \left|r_{n+3}\right| \leq\left(\frac{\left|\Gamma\left(2-\frac{2 \alpha}{3}+\frac{n \alpha}{3}\right)\right|}{\left|\Gamma\left(2-\frac{5 \alpha}{3}+\frac{n \alpha}{3}\right)\right|}\left|r_{n}\right|+\sum_{k=0}^{n}\left|r_{n-k+1}\right|\left|r_{k}\right|\right. \\
& \left.+\sum_{k_{1}=0}^{n} \sum_{k_{2}=0}^{k_{1}} \ldots \sum_{k_{q-1}=0}^{k_{q-2}}\left|s_{n-k+1}\right|\left|s_{k_{1}-k_{2}}\right| \ldots \mid s_{k_{q-2}-k_{q-1}|| s_{k_{q-1}} \mid}\right),
\end{aligned}
$$

and

$$
\left|s_{n+3}\right| \leq\left(\frac{\left|\Gamma\left(2-\frac{4 \alpha}{3 q}+\frac{n \alpha}{3}\right)\right|}{\left|\Gamma\left(2-\alpha-\frac{4 \alpha}{3 q}+\frac{n \alpha}{3}\right)\right|}\left|s_{n}\right|+\sum_{k=0}^{n}\left|s_{n-k+1}\right|\left|r_{k}\right|\right)
$$

Using the properties of $\Gamma$, we can easily show that $\frac{\left|\Gamma\left(2-\frac{2 \alpha}{3}+\frac{n \alpha}{3}\right)\right|}{\left|\Gamma\left(2-\frac{5 \alpha}{3}+\frac{n \alpha}{3}\right)\right|} \leq 1$ and $\frac{\left|\Gamma\left(2-\frac{4 \alpha}{3 q}+\frac{n \alpha}{3}\right)\right|}{\left|\Gamma\left(2-\alpha-\frac{4 \alpha}{3 q}+\frac{n \alpha}{3}\right)\right|}$ $\leq 1$

Therefore, we can write

$$
\begin{aligned}
& \left|r_{n+3}\right| \leq M\left(\left|r_{n}\right|+\sum_{k=0}^{n}\left|r_{n-k+1}\right|\left|r_{k}\right|\right. \\
& \left.+\sum_{k_{1}=0}^{n} \sum_{k_{2}=0}^{k_{1}} \ldots \sum_{k_{q-1}=0}^{k_{q-2}}\left|s_{n-k+1}\right|\left|s_{k_{1}-k_{2}}\right| \ldots \mid s_{k_{q-2}-k_{q-1}|| s_{k_{q-1}} \mid}\right),
\end{aligned}
$$

and

$$
\left|s_{n+3}\right| \leq N\left(\left|s_{n}\right|+\sum_{k=0}^{n}\left|s_{n-k+1}\right|\left|r_{k}\right|\right)
$$

where $M=\max \left(\left|\frac{1}{b_{1}}\right|,\left|\frac{a_{1}}{b_{1}}\right|,\left|\frac{c q}{b_{1}}\right|\right)$ and $N=\max \left(\left|\frac{1}{b_{2}}\right|,\left|\frac{a_{2}}{b_{2}}\right|\right)$ for an arbitrary $n$.

We can now define another power series form as

$$
K(\xi)=\sum_{n=0}^{\infty} y_{n} \xi^{n} \quad \text { and } \quad L(\xi)=\sum_{n=0}^{\infty} z_{n} \xi^{n}
$$


and let $y_{i}=\left|r_{i}\right|$ and $z_{i}=\left|s_{i}\right|, i=0,1, \ldots$. We can then easily obtain

$$
\begin{aligned}
& y_{n+3} \leq M\left(y_{n}+\sum_{k=0}^{n} y_{n-k+1} y_{k}\right. \\
& \left.+\sum_{k_{1}=0}^{n} \sum_{k_{2}=0}^{k_{1}} \ldots \sum_{k_{q-1}=0}^{k_{q-2}} z_{n-k+1} z_{k_{1}-k_{2}} \ldots z_{k_{q-2}-k_{q-1}} z_{k_{q-1}}\right),
\end{aligned}
$$

and

$$
z_{n+3} \leq N\left(z_{n}+\sum_{k=0}^{n} z_{n-k+1} y_{k}\right)
$$

Thus, it is obvious that $\left|y_{n}\right| \leq r_{n}$ and $\left|z_{n}\right| \leq s_{n}$ for $n=0,1,2, \ldots$. This also confirms that the series Equation (59) are the majorant series of Equation (44). We now have to prove that the series $K(\xi)$ and $L(\xi)$ have positive radius of convergence. By elementary calculations, we have the following:

$$
\begin{aligned}
& K(\xi)=y_{0}+y_{1} \xi+y_{2} \xi^{2}+y_{3} \xi^{3}+M\left(\sum_{n=0}^{\infty} y_{n}+\sum_{n=0}^{\infty} \sum_{k=0}^{n} y_{n-k+1} y_{k}\right. \\
& \left.+\sum_{n=0}^{\infty} \sum_{k_{1}=0}^{n} \sum_{k_{2}=0}^{k_{1}} \ldots \sum_{k_{q-1}=0}^{k_{q-2}} z_{n-k+1} z_{k_{1}-k_{2}} \ldots z_{k_{q-2}-k_{q-1}} z_{k_{q-1}}\right) \xi^{n+3},
\end{aligned}
$$

and

$$
L(\xi)=z_{0}+z_{1} \xi+z_{2} \xi^{2}+z_{3} \xi^{3}+N\left(\sum_{n=0}^{\infty} z_{n}+\sum_{n=0}^{\infty} \sum_{k=0}^{n} z_{n-k+1} y_{k}\right) \xi^{n+3}
$$

We can take into account an implicit functional system with respect to the independent variable $\xi$ as follows:

$$
\mathcal{K}(\xi, K)=K-y_{0}-y_{1} \xi-y_{2} \xi^{2}-y_{3} \xi^{3}-M\left(\xi^{2}\left(K-y_{0}\right)+\xi^{2} K\left(K-y_{0}\right)+\xi^{2} L\left(L-z_{0}\right)^{q-2}\right),
$$

and

$$
\mathcal{L}(\xi, L)=L-z_{0}-z_{1} \xi-z_{2} \xi^{2}-z_{3} \xi^{3}-N\left(\xi^{2}\left(L-z_{0}\right)+\xi^{2} L\left(K-y_{0}\right)\right) .
$$

It is clear that $\mathcal{K}$ and $\mathcal{L}$ are analytical in the neighborhood of $\left(0, y_{0}\right)$ and $\left(0, z_{0}\right)$. Therefore, $\mathcal{K}\left(0, y_{0}\right)=0, \mathcal{L}\left(0, z_{0}\right)=0$, and $\frac{\partial}{\partial K} \mathcal{K}\left(0, y_{0}\right) \neq 0, \frac{\partial}{\partial L} \mathcal{L}\left(0, z_{0}\right) \neq 0$ According to the implicit function theorem [43], the convergence is proved.

\section{Conclusions}

In this paper, we used a Lie point symmetry method in order to reduce a time fractional generalized coupled Drinfeld-Sokolov system to a time fractional coupled ODE system with the aid of the Riemann Liouville derivative and the fractional EK differential operator. By using the İbragimov conservation theorem, we obtained conservation vectors of the system. We then acquired explicit exact solutions of the reduced time fractional coupled ODE system by using a power series expansion method and proved that the series solutions are convergent.

Author Contributions: Methodology, writing—review and editing, S.G.; funding acquisition, S.W.Y.; formal analysis, M.I. All authors have read and agreed to the published version of the manuscript. 
Funding: The National Natural Science Foundation of China (No. 71601072), the Key Scientific Research Project of Higher Education Institutions in Henan Province of China (No. 20B110006), and the Fundamental Research Funds for the Universities of Henan Province.

Institutional Review Board Statement: Not applicable.

Informed Consent Statement: Not applicable.

Data Availability Statement: Not applicable.

Conflicts of Interest: The authors declare no conflict of interest.

\section{References}

1. Diethelm, K. The Analysis of Fractional Differential Equations; Springer: Berlin, Germany, 2010.

2. Miller, K.S.; Ross, B. An Introduction To the Fractional Calculus and Fractional Differential Equations; Wiley: New York, NY, USA, 1993.

3. Podlubny, I. Fractional Differential Equations; Academic Press: San Diego, CA, USA, 1999.

4. Oldham, K.B.; Spanier, J. The Fractional Calculus; Academic Press: San Diego, CA, USA, 1974.

5. Guy, J. Lagrange characteristic method for solving a class of nonlinear partial differential equations of fractional order. Appl. Math. Lett. 2006, 19, 873-880. [CrossRef]

6. El-Sayed, A.; Gaber, M. The Adomian decomposition method for solving partial differential equations of fractal order in finite domains. Phys. Lett. A 2006, 359, 175-182. [CrossRef]

7. Baleanu, D.; Inc, M.; Yusuf, A.; Aliyu, A.I. Institute of Space Sciences Lie symmetry analysis, exact solutions and conservation laws for the time fractional modified Zakharov-Kuznetsov equation. Nonlinear Anal. Model. Control. 2017, 22, 861-876. [CrossRef]

8. Jumarie, G. Cauchy's integral formula via the modified Riemann-Liouville derivative for analytic functions of fractional order. Appl. Math. Lett. 2010, 23, 1444-1450. [CrossRef]

9. Sardar, A.; Husnine, S.M.; Rizvi, S.T.R.; Younis, M.; Ali, K. Multiple travelling wave solutions for electrical transmission line model. Nonlinear Dyn. 2015, 82, 1317-1324. [CrossRef]

10. Ali, S.; Rizvi, S.T.R.; Younis, M. Traveling wave solutions for nonlinear dispersive water-wave systems with time-dependent coefficients. Nonlinear Dyn. 2015, 82, 1755-1762. [CrossRef]

11. Cheemaa, N.; Younis, M. New and more exact traveling wave solutions to integrable $(2+1)$-dimensional Maccari system. Nonlinear Dyn. 2016, 83, 1395-1401. [CrossRef]

12. Cheemaa, N.; Younis, M. New and more general traveling wave solutions for nonlinear Schrodinger equation. Waves Random Complex Media 2016, 26, 84-91. [CrossRef]

13. Deng, W. Finite Element Method for the Space and Time Fractional Fokker-Planck Equation. SIAM J. Numer. Anal. 2009, 47, 204-226. [CrossRef]

14. Zhang, S. Application of Exp-function method to a KdV equation with variable coefficients. Phys. Lett. A 2007, 365, 448-453. [CrossRef]

15. Zhang, X.; Zhao, J.; Liu, J.; Tang, B. Homotopy perturbation method for two dimensional time-fractional wave equation. Appl. Math. Model. 2014, 38, 5545-5552. [CrossRef]

16. Fadravi, H.H.; Nik, H.S.; Buzhabadi, R. Homotopy Analysis Method for Solving Foam Drainage Equation with Space- and Time-Fractional Derivatives. Int. J. Differ. Equ. 2011, 2011, 1-12. [CrossRef]

17. Gurefe, Y.; Sonmezoglu, A.; Misirli, E. Application of the trial equation method for solving some nonlinear evolution equations arising in mathematical physics. Pramana 2011, 77, 1023-1029. [CrossRef]

18. Zheng, B.; Wen, C. Exact solutions for fractional partial differential equations by a new fractional sub-equation method. Adv. Differ. Equ. 2013, 2013, 199. [CrossRef]

19. Kumar, S.; Kour, B.; Yao, S.-W.; Inc, M.; Osman, M. Invariance Analysis, Exact Solution and Conservation Laws of (2 + 1) Dim Fractional Kadomtsev-Petviashvili (KP) System. Symmetry 2021, 13, 477. [CrossRef]

20. Kour, B.; Kumar, S. Space time fractional Drinfel'd-Sokolov-Wilson system with time-dependent variable coefficients: Symmetry analysis, power series solutions and conservation laws. Eur. Phys. J. Plus 2019, 134, 1-15. [CrossRef]

21. Lukashchuk, S.Y. Symmetry reduction and invariant solutions for nonlinear fractional diffusion equation with a source term. Ufim. Mat. Zhurnal 2016, 8, 111-122. [CrossRef]

22. Lie, S. Theorie der Transformationsgruppen II; Written with the help of Friedrich Engel; BG Teubner: Leipzig, Germany, 1890.

23. Bluman, G.W.; Kumei, S. Symmetries and Differential Equations; Springer Science and Business Media: Berlin, Germany, 2013; Volume 81.

24. Ibragimov, N.K. Elementary Lie Group Analysis and Ordinary Differential Equations; Wiley: New York, NY, USA, 1999 ; Volume 197.

25. Clarkson, P.A.; Kruskal, M.D. New similarity reductions of the Boussinesq equation. J. Math. Phys. 1989, 30, 2201-2213. [CrossRef]

26. Clarkson, P.A. New similarity solutions for the modified Boussinesq equation. J. Phys. A Math. Gen. 1989, $22,2355-2367$. [CrossRef]

27. Noether, E. Invariant variation problems. Transp. Theory Stat. Phys. 1971, 1, 186-207. [CrossRef]

28. Atanacković, T.M.; Konjik, S.; Pilipović, S.; Simić, S. Variational problems with fractional derivatives: Invariance conditions and Noether's theorem. Nonlinear Anal. Theory Methods Appl. 2009, 71, 1504-1517. [CrossRef] 
29. Ibragimov, N.H. A new conservation theorem. J. Math. Anal. Appl. 2007, 333, 311-328. [CrossRef]

30. Lukashchuk, S.Y. Conservation laws for time-fractional sub diffusion and diffusion-wave equations. Nonlinear Dyn. 2015, 80, 791-802. [CrossRef]

31. Coclite, G.M.; Dipierro, S.; Maddalena, F.; Valdinoci, E. Singularity Formation in Fractional Burgers' Equations. J. Nonlinear Sci. 2020, 30, 1285-1305. [CrossRef]

32. Kirane, M.; Malik, S.A. The profile of blowing-up solutions to a nonlinear system of fractional differential equations. Nonlinear Anal. Theory Methods Appl. 2010, 73, 3723-3736. [CrossRef]

33. Miškinis, P. Some properties of fractional burgers equation. Math. Model. Anal. 2002, 7, 151-158. [CrossRef]

34. Sahoo, S.; Ray, S.S. Invariant analysis with conservation laws for the time fractional Drinfeld-Sokolov-Satsuma-Hirota equations. Chaos Solitons Fractals 2017, 104, 725-733. [CrossRef]

35. Jumarie, G. Modified Riemann-Liouville derivative and fractional Taylor series of nondifferentiable functions further results. Comput. Math. Appl. 2006, 51, 1367-1376. [CrossRef]

36. Gazizov, R.K.; A Kasatkin, A.; Lukashchuk, S.Y. Symmetry properties of fractional diffusion equations. Phys. Scr. 2009, T136, 14-16. [CrossRef]

37. Kiryakova, V.S. Generalized Fractional Calculus and Applications; CRC Press: Boca Raton, FL, USA, 1993.

38. Huang, Q.; Zhdanov, R. Symmetries and exact solutions of the time fractional Harry-Dym equation with Riemann-Liouville derivative. Phys. A Stat. Mech. Its Appl. 2014, 409, 110-118. [CrossRef]

39. Wang, G.-W.; Xu, T.-Z. Invariant analysis and exact solutions of nonlinear time fractional Sharma-Tasso-Olver equation by Lie group analysis. Nonlinear Dyn. 2013, 76, 571-580. [CrossRef]

40. Gazizov, R.; Ibragimov, N.; Lukashchuk, S. Nonlinear self-adjointness, conservation laws and exact solutions of time-fractional Kompaneets equations. Commun. Nonlinear Sci. Numer. Simul. 2015, 23, 153-163. [CrossRef]

41. Hashemi, M. Group analysis and exact solutions of the time fractional Fokker-Planck equation. Phys. A Stat. Mech. Its Appl. 2015, 417, 141-149. [CrossRef]

42. Nuseir, A.S.; Al-Hasson, A. Power series solution for nonlinear system of partial differential equations. Appl. Math. Sci. 2012, 6, 5147-5159.

43. Rudin, W. Principles of Mathematical Analysis; McGraw-Hill: New York, NY, USA, 1976; Volume 3. 